\title{
Case of Tuberculous Meningitis with Acute Presentation
}

\section{B. Shenoy ${ }^{1}$, B. Banerjee ${ }^{2}$, Archana . $M^{3}$. B. Bharath ${ }^{4}$ \\ Head of Paediatric Infectious Diseases, ${ }^{2}$ Paediatric Neurologist,}

Associate Consultant in Paediatric Infectious Diseases. ${ }^{4}$ Fellow in Paediatric Infectious Diseases.

Manipal Hospital, Bangalore, India.

\section{INTRODUCTION}

Tuberculous meningitis (TBM) is one of the common infections of central nervous system (CNS) in India. It poses challenges in diagnosis as symptoms can be nonspecific initially, there is no single diagnostic test with high sensitivity and microbiologic yield is low.

However, the outcome is directly dependent on the stage at which treatment is initiated. Early treatment can minimize complications, therefore attempts at early diagnosis with clinical suspicion and appropriate laboratory tests is warranted.

\section{CASE HISTORY}
A 7-year-old, presented with
* Fever since 2 days,
* Headache since 2 days and
* Generalized seizure on 2nd day of illness.

On evaluation child was febrile, BP 96/62 mm Hg, drowsy at admission, had diplopia though extra ocular movements were normal and had terminal neck stiffness.

\section{INVESTIGATION}

* Complete Blood counts were normal.

* CRP was negative.

* Child had hyponatremia $(127 \mathrm{mmol} / \mathrm{L})$ requiring correction.

* Contrast enhanced CT scan was normal.

* CSF analysis showed 2 lymphocytes, glucose of 68 $\mathrm{mg} / \mathrm{dl}$, protein of $10.4 \mathrm{mg} / \mathrm{l}$.

* Meningoencephalitis panel (PCR) was positive for TB, cultures were sterile.

\section{MANAGEMENT}

* Child received sodium correction in view of hyponatremia with seizures following which his sensorium rapidly improved.

* After PCR was positive for TB child was treated with 4 drug ATT with steroids.

* Child completed ATT with complete recovery.

\section{DISCUSSION}

* TB meningitis may present with a short febrile illness ( $<7$ days). The clues for suspicion would be presence of altered sensorium and focal neurologic signs like cranial nerve palsies.

* Hyponatremia in the setting of CNS infection may be a clue for considering TBM.

* CSF TB PCR is invaluable in making a diagnosis especially in cases with short or atypical history.

\section{KEYWORDS}

Tuberculous meningitis, acute presentation 